\title{
Switching attention within and between categories: Evidence for intracategory inhibition
}

\author{
W. TRAMMELL NEILL \\ University of South Florida, Tampa, Florida 33620
}

\begin{abstract}
In a variation of the Posner and Snyder (1975b) paradigm, subjects made speeded "same" or "different" responses to pairs of digits or letters on the basis of name identity (Experiment 1) or physical identity (Experiment 2). Each target pair was preceded by an informative (letter or digit) or noninformative (plus-sign) warning signal. The letter or digit represented by an informative warning signal had a high probability of appearing in the subsequent target pair (expected condition). On trials in which the expected stimulus did not appear in the target pair, the target pair belonged with equal probability to either the same category (either letters or digits) as the expected stimulus or to the opposite category (unexpected-similar and unexpected-opposite conditions, respectively). The pattern of "benefits" and "costs" of attentional expectancy found by Posner and Snyder for "same" responses was replicated: Subjects were faster and more accurate in the expected condition than in the neutral (uninformative warning signal) condition, but slower and less accurate in the unexpected conditions. Present theoretical interest concerns "same" response times and accuracy for the two unexpected conditions. Under instructions emphasizing accuracy as well as speed (Experiments 1 and 2), performance was worse in the unexpected-similar condition than in the unexpected-opposite condition. Only when instructions deemphasized accuracy (Experiment 2) was performance better in the unexpected-similar condition than in the unexpected-opposite condition. The fact that subjects may have more difficulty switching attention within a category than between categories (at least when instructions emphasize accuracy as well as speed) contrasts sharply with current theoretical emphasis on intracategorical facilitation effects in the priming paradigm. The results are interpreted within the framework of a model of attention (Keele \& Neill, 1978) in which the activation of associates to the focus of attention is actively and optionally suppressed so as to reduce present or potential interference with the focal information processing.
\end{abstract}

A wide range of studies have demonstrated that recent access to a semantic category can facilitate subsequent retrieval from that category. The importance of category access in recall of organized lists is well established (e.g., Tulving \& Pearlstone, 1966). Facilitatory effects of associative relationships have more recently been explored in several reaction time paradigms. Loftus (1973) found that the time to generate the name of an exemplar of a given category is reduced if the subject has just named another exemplar of the same category. Meyer and Schvaneveldt (1971) demonstrated that lexical decision (word-nonword judgment) is faster when the target word is associatively related to the target judged on the preceding trial than when unrelated. While such facilitation does not

Part of this research was presented to a meeting of the Southeastern Psychological Association in Atlanta, Georgia, in March 1978. The experiments comprise part of a dissertation oresented to the University of Oregon in partial fulfillment of the requirements for the degree of Doctor of Philosophy. I am ndebted to Steven Keele, Michael Posner, Ray Hyman, and Arthur Farley for guidance and encouragement. Extensive sommentary from an anonymous reviewer greatly facilitated evision of the manuscript. Requests for reprints should be sent :o W. Trammell Neill, Department of Psychology, University of jouth Ilorida, Tampa, Florida 33620. necessarily require conscious expectation of a semantic relationship (Fischler, 1977), conscious attention has also been found to influence semantic facilitation (Neely, 1976, 1977).

While specific accounts of intracategory facilitation may differ, there seems to be a general agreement that members of a category are somehow more highly associated, and that interitem accessibility should increase directly with categorical similarity (see, e.g., Collins \& Loftus, 1975; Schvaneveldt \& Meyer, 1973). However, it should be noted that recent access to a category does not always facilitate subsequent processing of other members of that category. Indeed, there are cases in which the opposite effect, interference, seems to occur. Rosch (1975a) describes a series of experiments in which a "priming" category name was presented before a pair of words or pictures to be judged as "same" or "different." Consistent with other semantic facilitation effects, category and physical identity matches of "good" (high-typicality) instances of the primed category were faster than when no category was primed. Similar results were found for color matches primed by color category names (Rosch, 1975b). Yet, in both studies, physical identity matches of "poor" (lowtypicality) exemplars were actually hampered by 
presentation of the category name. This result was not simply due to poor exemplars' not really belonging to the assigned category, since pairs drawn from a category other than the primed category did not produce the same pattern of results. Thus, category priming appears to have made high-typicality instances more accessible at a cost to low-typicality instances.

Another case of apparent intracategory inhibition appears in recall of categorized lists. While use of category names as cues facilitates recall (Tulving \& Pearlstone, 1966), the addition of list items as cues actually depresses recall of the remaining items from the same category (Roediger, 1973, 1974; Rundus, 1973; Slamecka, 1968, 1969). Watkins (1975) has shown that recall of list items can similarly be depressed by extralist cues belonging to the same category as the target words. Again, increasing the accessibility of certain category instances seems to be at a cost to the accessibility of other members of the same category.

Why does categorical similarity yield facilitation in some tasks but interference in others? Keele and Neill (1978) propose a model of attention in which a stimulus automatically activates not only its own representation in memory but a variety of featurally and associatively related memories as well. Attention may then be required to narrow down the range of activated memory structures to those specifically appropriate to the task. In other words, while "recognition" in the sense of memory activation may occur without attention, "identification" in the sense of unique specification may not. (See also the "verification" model proposed by Becker, 1976.) Failure to uniquely identify a presented word may result in judgments based on other related information, which may sometimes be appropriate to the task and sometimes not. As an extreme example, a semantic dyslexic may read the word "speak" as "talk," even though the printed word remains visible (Marshall \& Newcombe, 1973; Patterson \& Marcel, 1977; Shallice \& Warrington, 1975). The word has been "recognized," or else the subject would not report the associated word; yet, the subject is unable to uniquely identify the presented word. Allport (1977) and Marcel (in press; Marcel \& Patterson, 1978) both report similar phenomena in normal subjects with brief tachistoscopic word presentation.

The model of attention proposed by Keele and Neill (1978) goes further to suggest that direct suppression may play an important role in the "narrowing-down" process, such that the activation of irrelevant and potentially conflicting information is reduced. The reduction of activation can be (but is not necessarily) so extreme as to reduce retrievability to below the "baseline" level prior to the recent activation. Direct evidence for a mechanism of selective inhibition was provided by Neill (1977b) in a version of the Stroop (1935) color-word task. In this task, subjects must name the color (e.g., red) of a printed word that itself names a different color (e.g., "green"). Neill found that color naming latency was actually longer if the response had been recently activated by the distractor word appearing on the preceding trial. This implies that the activated competing response had been selectively inhibited on the preceding trial, resulting in reduced retrievability as a response on the subsequent trial.

According to the Keele and Neill (1978) analysis, intracategory facilitation may be more likely in tasks that require a lesser degree of unique stimulus identification. For example, in the lexical decision task (e.g., Meyer \& Schvaneveldt, 1971; Neely, 1976, 1977), activation of a set of associates may be sufficient to judge the stimulus as a word; it is not necessary to uniquely identify the word by narrowing down that set. Thus, those associates should remain relatively more retrievable over subsequent trials. Similarly, if one is required to name an instance of a category (Loftus, 1973), there are no constraints on which exemplar to name (except, perhaps, to avoid repetition). In contrast, recall of a memory list requires retrieval of specific, uniquely identified words, not just any associates of the original stimuli. Maintaining the activation of "irrelevant" category members may then prevent unique specification of the target memory. Matching tasks requiring judgment of "same" or "different" (as in Rosch, 1975a, 1975b) might also be disrupted by activating a large set of associates if those associates include possible stimulus alternatives. For example, if the letter $A$ associatively activates $B$, then the pair AA might be spuriously mismatched as $A B$. If, however, attention has been previously drawn to $\mathrm{A}$, it may be possible to circumvent some interference by suppressing the activation of $\mathrm{B}$. Of course, if $\mathrm{BB}$ were then unexpectedly presented, the match might be very difficult-perhaps more so than a match involving some pair unrelated to A, such as "22." This is, in fact, the crux of the experiments to be reported.

Existing evidence for intracategory inhibition is rather sparse, despite its plausibility. While part-list cuing effects in recall are well documented, a variety of explanations have also been proposed (see Basden, Basden, \& Galloway, 1977; Roediger, Stellon, \& Tulving, 1977; and previously cited references). While such explanations are not necessarily incompatible with the present model, the analogy between free recall and previously discussed reaction time paradigms may seem strained to some. The category-priming inhibition of low-typicality pairs is consistent over several experiments by Rosch (1975a, 1975b), but it might be discounted as an isolated phenomenon, limited to lowtypicality pairs with category priming. It is especially disturbing that the result only occurred with physical identity matches and not with category identity matches. Thus, the major thrust of the experiments to be reported is to determine whether intracategory inhibition can be demonstrated under conditions for which there is some a priori theoretical expectation of its occurrence, that is, a matching task in which 
successful performance depends on directing attention to specific members of two well defined categories, letters and digits.

The present experiments employed a variation of the expectancy-priming paradigm developed by Posner and Snyder (1975a, 1975b). Posner and Snyder instructed subjects to judge pairs of letters as the "same" or "different." Each pair was preceded by a warning signal that could be either a letter or a neutral "plus" sign. In the condition of most interest, a letter used as a warning signal had a high probability $(.80)$ of also appearing in the subsequent target pair, thereby serving to direct attention to that letter. As expected, pairs containing the expected letter were matched faster and more accurately than pairs preceded only by a neutral plus sign. More important, pairs not containing the expected letter were matched more slowly and less accurately than in the neutral unprimed condition. Posner and Snyder interpreted this "cost" in reaction time and errors as reflecting the difficulty of reallocating attention to the unexpected event.

The Posner and Snyder (1975a, 1975b) paradigm was extended here to include two categories of target stimuli, a set of letters (A-H) and a set of digits (1-8). When the expected character was not present in the target pair, other members of either category set were squally likely to appear. If attention switching is facilitated by categorical similarity (as suggested, for zxample, by lexical decision studies), "same" matches of unexpected stimuli from the same category as the unexpected stimulus should be more efficient than "same" matches of unexpected stimuli from the spposite category. On the other hand, if intracategory inhibition occurs (as suggested, perhaps, by Rosch, $1975 a, 1975 b$ ), such suppression may hamper samezategory matches more than opposite-category matches.

\section{EXPERIMENT 1}

\section{Method}

Subjects. Twelve University of Oregon undergraduates (five male, seven female) were each paid $\$ 5$ for participating in two individual 1 -h sessions, usually on successive days. Most subjects lad previously participated in other reaction time experiments.

Apparatus. Letter and digit stimuli were generated on a Jewlett-Packard 1300A X-Y display oscilloscope. A PDP-15 :omputer controlled stimulus presentation and recorded latency lata from two microswitch response keys.
Procedure. Each trial consisted of a 400-msec warning signal, followed by a 100 -msec blank period, and then a pair of letters or digits that remained visible until the subject's response. In a block of 128 trials, the warning signal was drawn randomly from the set of capital letters A-H on 48 trials and from the set of digits 1.8 on 48 trials. On the remaining 32 trials, the warning signal was a simple plus sign ("+"). When a letter or digit appeared as the warning signal, the subsequent test pair was chosen to include the warning signal character on $66.7 \%$ of such trials. When the warning signal was not chosen for the test pair, any other letter or digit (from either stimulus set) was equally likely to appear. Thus, in a block of 128 trials, 64 target pairs included an expected letter or digit (expected condition), 16 target pairs were unexpected but drawn from the same category as the expected stimulus (unexpected-similar condition), 16 target pairs were unexpected and drawn from the category opposite to the expected stimulus (unexpectedopposite condition), and 32 target pairs were preceded only by the noninformative plus sign (neutral condition). In half the test pairs for each condition, the two characters were identical in name ("same" trials); in other half, a letter was paired with a different letter, or a digit with another digit ("different" trials). Half the letter pairs were presented in all capitals (e.g., AA or $A B)$; in the other half, the second letter was presented in lowercase (e.g., $\mathrm{Aa}$ or $\mathrm{Ab}$ ).

Subjects responded "same" or "different" by pressing response keys under the left and right index fingers, respectively. They were instructed to use the warning signal to anticipate the content of the test pair, as this would facilitate responding on a majority of the trials. Additional instructions emphasized that the stimuli were to be matched according to name, not physical appearance. Subjects were told to respond as quickly as possible, but to keep errors to a minimum.

In each session, subjects received one practice block followed by eight experimental blocks. Subjects initiated each block at will by pressing a response key while "READY" was displayed on the oscilloscope.

\section{Results and Discussion}

The results of Experiment 1 are displayed in Table 1. The basic "costs" and "benefits" of attention described by Posner and Snyder (1975a, 1975b) are clearly replicated in the "same" trial data. Expected pairs yielded "benefit" relative to unprimed (neutral) pairs in both reaction time $[\mathrm{t}(11)=10.03, \mathrm{p}<.001]$ and accuracy $[t(11)=2.30, p<.05]$. Unexpected-similar pairs produced "cost" relative to the neutral condition in both reaction time $[\mathrm{t}(11)=9.58, \mathrm{p}<.001]$ and accuracy $[\mathrm{t}(11)=7.29, \mathrm{p}<.001]$. Unexpected-opposite pairs also produced "cost" relative to the neutral condition in both reaction time $[\mathrm{t}(11)=8.98, \mathrm{p}<.001]$ and errors $[t(11)=5.95, p<.001]$. (All reported $t$ tests are two-tailed.)

Table 1

Experiment 1: Mean Reaction Times (RT) in Milliseconds and Error Proportions (E) as a Function of Expectancy Condition and Category Relation

\begin{tabular}{|c|c|c|c|c|c|c|}
\hline \multirow{2}{*}{$\begin{array}{c}\text { Expectancy Condition and } \\
\text { Category Relation } \\
\end{array}$} & \multicolumn{3}{|c|}{ "Same" Trials } & \multicolumn{3}{|c|}{ "Different" Trials } \\
\hline & Example & RT & $\mathrm{E}$ & Example & RT & E \\
\hline Expected & A-AA, A-Aa, 1-11 & 401 & .04 & $A-A B, A-A b, 1-12$ & 465 & .08 \\
\hline Neutral & $+-\mathrm{AA},+-\mathrm{Aa},+-11$ & 468 & .06 & $+-A B,+-A b,+-12$ & 495 & .07 \\
\hline Unexpected-Similar & $\mathrm{A}-\mathrm{BB}, \mathrm{A}-\mathrm{Bb}, 1-22$ & 517 & .24 & $A-B C, A-B c, 1-23$ & 460 & .05 \\
\hline Unexpected-Opposite & A-22, 1-AA, 1-Aa & 514 & .19 & $1-\mathrm{BC}, 1-\mathrm{Bc}, \mathrm{A}-23$ & 467 & .05 \\
\hline
\end{tabular}

Vote-In the examples, the character to the left of the hyphen denotes the warning signal, and the pair to the right of the hyphen 'enotes the pair to be judged as "same" or "different." 
The critical comparison in this experiment is between the unexpected-similar and the unexpected-opposite conditions. While "same" reaction times did not differ between these two conditions, the error rates were significantly different in the direction consistent with intracategory inhibition $[t(11)=2.78, p<.02]$. In other words, subjects appeared to have more difficulty switching attention within a category than switching attention between categories.

No comparable differences were obtained between unexpected-similar and unexpected-opposite pairs requiring a "different" response, nor were any specifically predicted. In the expected condition, comprising a majority of all trials, the difficulty of retrieving or allocating attention to the second character relative to the first (expected) character is by itself a valid cue for responding "different." Thus, if time to reallocate attention has any influence on the matching decision, any benefit of switching attention more rapidly from the expected to the unexpected stimulus might be offset by a bias to respond "same"; conversely, a detriment to switching might be compensated by a bias to respond "different." In "same" trials, however, both factors would be expected to operate in the same direction. Since the present experiments are not designed to separate retrieval and comparison processes, predictions are safely limited to "same" trials.

It should be noted in passing that Posner and Snyder (1975b) found "different" responses to be slower in the expected condition than the neutral condition, whereas a difference in the opposite direction appears here. Posner and Snyder suggest that their results are contaminated by a strategy of matching the pair letters with the priming stimulus. However, the reported difference is small and does not consistently appear in similar experiments (Posner, Note 1). The "matching-toprime" strategy also predicts that "different" responses in the unexpected conditions should be faster than in the neutral condition. In this respect, the present data do suggest a matching-to-prime strategy. Given the difficulty of interpreting "different" trials, the remaining discussion will focus on "same" trials, although the "different" trial data are presented for the interested reader. Note that a matching-to-prime strategy does not vitiate the present theoretical focus; namely, the fact that unexpected-similar pairs are matched less efficiently (with each other or with the priming stimulus) than unexpected-opposite pairs is still indicative of intracategory inhibition.

The fact that intracategory inhibition was manifested in error rate only and not reaction times was unexpected. This may reflect a self-imposed response deadline on the part of the subject (see, e.g., Krueger, 1978). Subjects' overall reaction times are much faster than on the minority of trials in which unexpected stimuli only are presented. On the latter trials, subjects may tend to sacrifice some accuracy in order to maintain the overall set for rapid responding, hence the relatively high error rates in these conditions despite instructional emphasis on accuracy as well as speed. This explanation is, however, entirely post hoc and must await future testing.

The intracategory inhibition obtained for "same" responses was manifested equally in the error rates for letter pairs and digit pairs, and it occurred for physically identical matches (digits and same-case letters) as well as for name-only matches (opposite-case letters). (Detailed analyses are available in Neill, 1977a.) Unfortunately, a name identity-match condition was not available for digits. While it seems unlikely that the asymmetry of match levels for letters and digits could have played a crucial role in producing the intracategory inhibition effect, the potential theoretical importance of this effect warranted a second experiment employing a more balanced design in which this asymmetry of match levels was not present.

\section{EXPERIMENT 2}

Match levels were equated for letters and digits in Experiment 2 by requiring matches for both sets to be made only on the basis of physical identity. While digits were presented as in Experiment 1, letter pairs included only capital letters. A further change was that the physical similarity of the warning signal to the target pair stimuli was deliberately reduced by using lowercase letters and digit names (e.g., "one") as priming stimuli. This modification was expected to minimize the likelihood that subjects would adopt the previously discussed strategy of matching test stimuli to the warning signal, although evidence for such a strategy in Experiment 1 was equivocal. In order to accommodate longer reading times for digit names, the total priming interval was increased to $750 \mathrm{msec}$ (from $500 \mathrm{msec}$ in Experiment 1).

A new manipulation introduced in Experiment 2 was to vary instructional emphasis on speed vs. accuracy. Category effects in an earlier version of these experiments were strongly correlated with individual differences in speed-accuracy tradeoff (Neill, 1977a). In addition, Neill (1977b) found suppression of the distractor response in the Stroop color-word task to be sensitive to subtle changes in experimental procedure. In particular, it was suggested that degree of suppression varies with the relative demands for speed vs. accuracy. In terms of the present model, the narrowing-down process would necessarily be more complete (and take more time) under strict accuracy demands. When speed is emphasized at some cost to accuracy, less narrowing down may be required, leaving associates to the attended stimulus relatively more activated. Accordingly, it was predicted that performance would be worse in the unexpectedsimilar condition than in the unexpected-opposite condition (intracategory inhibition) under instructions 
zmphasizing accuracy. However, unexpected-similar performance might be better than unexpected-opposite when speed was emphasized at some cost to accuracy.

\section{Method}

Subjects. Eight University of Oregon undergraduates (two nale, six female) who had not participated in Experiment 1 were zach paid $\$ 5$ for participating in two individual $1-\mathrm{h}$ sessions, usually on successive days. Most had previously participated in sther reaction time experiments.

Procedure. Each trial consisted of a 500 -msec warning signal, ollowed by a $250-\mathrm{msec}$ blank period, and then a target pair of sapital letters or digits. In a block of 100 trials, the warning iignal was drawn randomly from the set of lowercase letters 1 -h on 40 trials, and from the set of digit names "one" through "eight" on 40 trials. On the remaining 20 trials, the warning ignal was a neutral plus sign ("+"). When a lowercase letter or 1 digit name appeared as the warning signal, the capital letter or ligit of the same name as the warning signal appeared in the iubsequent target pair on $80 \%$ of such trials. On trials in which :he expected stimulus was not chosen for the target pair, any ther digit or letter (from either category set) was equally likely :o appear in the pair, with the restriction that letters were saired only with letters, and digits only with digits (as in Experiment 1). Thus, in a block of 100 trials, 64 target pairs ncluded the expected stimulus (expected condition), 8 pairs vere wholly unexpected but from the expected category unexpected-similar), 8 pairs were unexpected and from the spposite category (unexpected-opposite), and 20 pairs were receded only by the noninformative plus sign (neutral zondition). Half the pairs in each condition were composed of dentical characters ("same" trials); the other half paired lissimilar characters ("different" trials).

In each session, subjects received one practice block followed गy 10 experimental blocks. In one session, subjects were nstructed to respond as quickly as possible, sacrificing some saution for speed, but not making more than $10-15$ errors per slock ("speed" instructions). In the other session, subjects were nstructed to respond quickly but with the restriction that they make as few errors as possible ("accuracy" instructions). Half the subjects received speed instructions in the first session, and half received accuracy instructions in the first session.

Other aspects of procedure were similar to Experiment 1.

\section{Results and Discussion}

The results of Experiment 2 are displayed in Table 2. The instructions were markedly successful in manipuating speed-accuracy tradeoff. Under speed instructions, nean reaction time for correct responses was $366 \mathrm{msec}$, with an error proportion of .18. Under accuracy nstructions, mean correct reaction time was $413 \mathrm{msec}$, with an error proportion of .08 . While the latter error rate is still "high" by some standards, all subjects were faster and made more errors under speed instructions than under accuracy instructions.

Again, the basic pattern of attentional "costs" and "benefits" described by Posner and Snyder (1975a, 1975b) was replicated in "same" reaction times and accuracy. All comparisons of expected and unexpected condition "same" trials with the neutral condition were significant by $t$ test at the .01 level or better for both reaction times and errors, with one exception: The average reaction time for the unexpected-similar condition did not differ from the neutral condition under speed instructions. However, considering the rather large error difference (.19), it would be inappropriate to conclude that attentional "cost" did not occur for the unexpected-similar condition under speed instructions. Note that average reaction times even under accuracy instructions were faster than in Experiment 1, presumably reflecting the fact that physical identity matches are made more rapidly than name identity matches (Posner \& Mitchell, 1967).

The data obtained under accuracy instructions replicate the important finding of Experiment 1: While reaction times did not differ between unexpected-similar and unexpected-opposite "same" trials, error rates did differ significantly $[t(7)=4.65, p<.01]$. Error differences for all eight subjects were consistent with this pattern of intracategory inhibition.

A rather different pattern of results was obtained under speed instructions. Here, the error rates did not differ between unexpected-similar and unexpectedopposite conditions. However, reaction times were significantly faster for the unexpected-similar condition than for the unexpected-opposite condition $[\mathrm{t}(7)=3.55$, $p<.01]$. Reaction time differences under speed instructions for seven of the eight subjects were consistent with this pattern, the one reversal being rather small in magnitude $(8 \mathrm{msec})$. It is also notable that all seven consistent subjects showed a greater similar over opposite-category superiority under speed instructions than under accuracy instructions. Closer inspection of the data revealed that the one inconsistent subject performed at or below chance in several of the unex-

Table 2

Experiment 2: Mean Reaction Times (RT) in Milliseconds and Error Proportions (E) as a Function of Expectancy Condition, Category Relation, and Instructional Emphasis

\begin{tabular}{|c|c|c|c|c|c|c|c|c|c|c|}
\hline \multirow{4}{*}{$\begin{array}{l}\text { Expectancy Condition } \\
\text { nd Category Relation }\end{array}$} & \multicolumn{5}{|c|}{ "Same" Trials } & \multicolumn{5}{|c|}{ "Different" Trials } \\
\hline & \multirow[b]{3}{*}{ Example } & \multicolumn{4}{|c|}{ Instructions } & & \multicolumn{4}{|c|}{ Instructions } \\
\hline & & \multicolumn{2}{|c|}{ Speed } & \multicolumn{2}{|c|}{ Accuracy } & \multirow[b]{2}{*}{ Example } & \multicolumn{2}{|c|}{ Speed } & \multicolumn{2}{|c|}{ Accuracy } \\
\hline & & RT & $\mathrm{E}$ & RT & $\mathrm{E}$ & & RT & $\mathrm{E}$ & RT & E \\
\hline ixpected & a-AA, two- 22 & 334 & .11 & 369 & .05 & $a-A B$, two -23 & 373 & .23 & 408 & .08 \\
\hline Jeutral & $+-\mathrm{AA},+-22$ & 376 & .19 & 417 & .08 & $+-A B,+-23$ & 376 & .14 & 420 & .07 \\
\hline Jnexpected-Similar & $\mathrm{a}-\mathrm{BB}$, one- 22 & 369 & .38 & 437 & .28 & $\mathrm{a}-\mathrm{BC}$, one- 23 & 363 & .14 & 395 & .03 \\
\hline Jnexpected-Opposite & $\mathrm{a}-22$, one- $\mathrm{BB}$ & 395 & .38 & 442 & .22 & $\mathrm{a}-23$, one-AB & 355 & .10 & 403 & .06 \\
\hline
\end{tabular}

Vote-In the examples, the character to the left of the hyphen denotes the warning signal, and the pair to the right of the hyphen 'cnotes the pair to be judged as "same" or "different." 
pected conditions. The same subject showed a relatively large reaction time difference between the unexpectedsimilar and unexpected-opposite conditions under accuracy instructions. As a result, the trend toward a Category by Instruction interaction did not achieve significance in an analysis of variance performed on unexpected "same" trial data $[F(1,7)=3.69$, n.s. $]$. Main effects of instruction $[F(1,7)=6.70, p<.05]$ and of category $[F(1,7)=19.02, p<.01]$ were obtained. A more powerful analysis of variance in which response ("same" or "different") was included as a factor did, however, yield a significant Category by Instruction by Response interaction $[F(1,7)=7.18, p<.05]$, reflecting a Category by Instruction interaction trend for "same" trials but not for "different" trials. (Details may be found in Neill, 1977a.)

The data of Experiment 2 are consistent with the proposed model of intracategory inhibition. Under conditions emphasizing accuracy, subjects appear to suppress categorical associates of the expected stimulus, presumably to minimize potential competition. As in Experiment 1, this effect was manifested primarily in the error rates. However, under conditions demanding more speed at some cost to accuracy, categorical associates are left relatively unsuppressed and therefore are more easily retrieved. The intracategory superiority effect was manifested in reaction time instead of errors. Since the average response latency here was much shorter than under accuracy instructions in this experiment or in Experiment 1, a self-imposed "deadline" for response (such as discussed in Experiment 1) may have left more "room" for effects to appear in reaction time rather than errors. Again, however, this explanation is wholly post hoc and must be considered purely speculative.

\section{GENERAL DISCUSSION}

The most important result of Experiments 1 and 2 is the demonstration of intracategory inhibition. That is, under some conditions, it is more difficult to switch attention to an unexpected event in the same category as the expected event, than to an unexpected event in a different category. This result is surprising in the context of current theoretical emphasis on the facilitatory effects of categorical similarity (e.g., Collins \& Loftus, 1975; Neely, 1976, 1977; Schvaneveldt \& Meyer, 1973). It is consistent, however, with the existence of a selective inhibitory mechanism capable of suppressing the "noise" of activated associates to the attended event.

Two disclaimers are necessary regarding this conclusion. First, it is not likely that facilitatory and inhibitory processes are mutually exclusive. Rather, the observed category effect probably reflects a balance between coexistent underlying inhibitory and facilitatory processes. Indeed, it is proposed that intracategory inhibition is superimposed on a prior "spread of activation" (e.g., Collins \& Loftus, 1975;
Neely, 1976, 1977; Schvaneveldt \& Meyer, 1973) to categorical associates to the attended stimulus. The balance between inhibitory and facilitatory effects appears to be affected by external variables. Thus, in Experiment 2, inhibitory effects of categorical similarity outweighed facilitatory effects under instructions emphasizing accuracy; however, under instructions emphasizing speed, facilitatory effects apparently outweighed inhibitory effects. This is consistent with the hypothesis that the degree of selective inhibition depends on the relative need to uniquely identify the presented stimuli, according to the accuracy demands of the task.

Second, the mechanism responsible for intracategory inhibition probably does not account for all "inhibitory" effects of attention. This point may be illustrated by considering what may be involved in "switching attention." Posner and Snyder (1975a, $1975 b$ ) identify "time to switch attention" with the reaction time "cost" to matching two misprimed stimuli, relative to a neutral, unprimed condition. However, LaBerge (1973) suggests four likely components of switching time, which may be paraphrased as follows: (1) time to signal the switching mechanism, (2) time to "disengage" attention from the expected event (the "origin"), (3) time to "engage" attention with the unexpected event (the "destination"), (4) "transit" time between the "origin" and the "destination." Note that any or all of these components could also be present to some extent in the neutral, unprimed condition. Thus, reaction time "cost" should be viewed as a relative measure, rather than an absolute measure of switching time. More important, it is likely that these components are sensitive to different factors. For example, it is possible that intracategory inhibition affects time to signal the switching mechanism or to engage attention with the suppressed stimulus, but does not affect "transit" time between the expected and unexpected events. In fact, the spatial metaphor common in theories of semantic memory (e.g., Anderson, 1976; Collins \& Loftus, 1975; Schvaneveldt \& Meyer, 1973) suggests the latter component might always be favored by categorical similarity, which reinforces the previous point that the observed category effect may reflect a mixture of inhibitory and facilitatory effects.

The relative time courses of inhibitory and facilitatory category effects remain to be investigated, although it may prove difficult to separate them from the overall inhibitory and facilitatory effects of atten. tion whose time courses were extensively investigated by Posner and Snyder (1975a, 1975b). We may speculate from the current model and the results of Posner and.Snyder that intracategory facilitation from an automatic "spread of activation" would appear relatively early, followed by intracategory inhibition resulting from attentional suppression. It may be noted that the priming interval in Experiment 1 was $500 \mathrm{msec}$, while in Experiment 2 it was extended to $750 \mathrm{msec}$. The 
degree of intracategory inhibition was markedly similar for Experiment 1 (error proportion difference $=.05$ ) and Experiment 2 under accuracy instructions (error proportion difference $=.06$ ). However, the results of Posner and Snyder suggest that attentional inhibition is fully established by around $300 \mathrm{msec}$, and so it is not surprising that the effect did not differ between the two experiments. The average reaction time cost under accuracy instructions in Experiment 2 (23 msec) is only half that obtained under similar instructions in Experiment 1 ( $47 \mathrm{msec}$ ), but this difference is offset by an increase in error cost and is difficult to interpret.

Whether the present category effects with digits and letters can be generalized to more complex semantic categories also remains to be investigated. The categorical distinction between digits and letters has. however, played an important role in the development of contemporary theories of attention. Broadbent (1971) has argued that the selection of information by physical attributes ("stimulus set") is generally more efficient than selection by meaning ("response set"), favoring the operation of a selective attention mechanism at a stage of processing prior to stimulus categorization. Comparisons of stimulus set with response set have frequently relied on the digit-letter category distinction. For example, Sperling (1960) found a partial report superiority for seiecting iconic information by spatial location but not for selection by category (digits vs. letters). In dichotic listening studies, selection of ear of entry has been similarly compared with selection by digit vs. letter categories (e.g. Broadbent \& Gregory, 1964). A possible implication of the present results is that the superiority of stimulus set over response set, when it occurs, does not necessarily reflect the operation of a precategorical "filter." It may instead reflect the existence of intracategory rivalry at or beyond the stage of recognition.

The existence of intracategory inhibition has important implications for theories of both attention and semantic memory. Perhaps the broadest implication concerns the most basic nature of attention. Intracategory inhibition, as well as previously demonstrated selective inhibition effects (Neill, 1977b), is inconsistent with the conception of attention as simply the direct allocation of some unitary facilitatory resource or capacity. More recent theories of attention (c.g., Keele \& Neill, 1978; Posner \& Snyder, 1975a; Shaffer, 1975; Shiffrin \& Schneider, 1977) have begun to shift emphasis from the concept of "capacity" per se to the concept of "cognitive control." Accordingly, selective attention probably involves control mechanisms capable of manipulating stimulus information in a varicty of ways, including retrieval, rehearsal, elaboration, integration with other information, translation into response, or suppression. Human information processing is necessarily complex; it should not be surprising that control of that processing, selective attention, is complex as well.

\section{REFERENCE NOTE}

1. Posner, M. I. Personal communication, 1977.

\section{REFERENCES}

Allport, D. A. On knowing the meaning of words we are unable to report. In S. Dornic (Ed.), Attention and performance VI. Hillsdale, N.J: Erlbaum, 1977.

Anderson, J. R. Language, memory, and thought. Hillsdale, N.J: Erlbaum, 1976.

Basden, D. R., Basden, B. H., \& Galloway, B. C. Inhibition with part-list cuing: Some tests of the item strength hypothesis. Journal of Experimental Psychology: Human Learning and Memory, 1977, 3, 100-108.

BECKER, C. A. Allocation of attention during visual word recognition. Journal of Experimental Psychology: Human Perception and Performance, 1976, 2, 556-566.

Broadbent, D. E. Decision and stress. New York: Academic Press, 1971.

Broadbent, D. E., \& Gregory, M. Stimulus set and response set: The alternation of attention. Quarterly Journal of Experimental Psychology, 1964, 16, 309-317.

Collins, A. M., \& Loftus, E. F. A spreading-activation theory of semantic processing. Psychological Review, 1975, 82, 407-428.

Fischler, I. Associative facilitation without expectancy in a lexical decision task. Journal of Experimental Psychology: Human Perception and Performance, 1977, 3, 18-26.

KeELE, S. W., \& NeILL, W. T. Mechanisms of attention. In E. C. Carterette \& P. Friedman (Eds.), Handbook of perception (Vol. 9). New York: Academic Press, 1978.

Krueger, L. E. A theory of perceptual matching. Psychological Review, 1978, 85, 278-304.

LABERGE, D. Identification of two components of the time to switch attention: A test of a serial and a parallel model of attention. In S. Kornblum (Ed.), Attention and performance IV. New York: Academic Press, 1973.

Loftus, E. F. Activation of semantic memory. American Journal of Psychology, 1973, 86, 331-337

MARCEL., A. J. Unconscious reading: Experiments on people who do not know that they are reading. Visible Language, in press.

Marcel, A. J., \& Patterson, K. E. Word recognition and production: Reciprocity in clinical and normal studies. In J. Requin (Ed.), Attention and performance VII. Hillsdale, N.J: Erlbaum, 1978 .

Marshall, J. C., \& Newcombe, F. Patterns of paralexia: A psycholinguistic approach. Journal of Psycholinguistic Research, 1973, 2, 175-199.

Meyer, D. E., \& Schvaneveldt, R. W. Facilitation in recognizing pairs of words: Evidence of a dependence between retrieval operations. Journal of Experimental Psychology, 1971, 90, 227-234.

NeELY, J. H. Semantic priming and retrieval from lexical memory: Evidence for facilitatory and inhibitory processes. Memory \& Cognition, 1976, 4, 648-654.

NeEcy, J. H. Semantic priming and retrieval from lexical memory: Roles of inhibitionless spreading activation and limited-capacity attention. Journal of Experimental Psychology: General, 1977, 106, 226-254.

NeItl. W. T. Attention: The coordination of internal codes. Doctoral dissertation, University of Oregon, 1977. (Xerox University Microfilms No. 78-2551) (a)

NeILL, W. T. Inhibitory and facilitatory processes in selective attention. Journal of Experimental Psychology: Human Perception and Performance, 1977, 3, 444-450. (b)

Patterson, K. E., \& Marcel, A. J. Aphasia, dyslexia and the phonological coding of written words. Quarterly Journal of Experimental Psychology, 1977, 29, 307-318.

Posnef, M. I., \& Mitchell, R. F. Chronometric analysis of classification. Psychological Review, 1967, 74, 392-409. 
Posner, M. I., \& Snyder, C. R. R. Attention and cognitive control. In R. L. Solso (Ed.), Information processing and cognition: The Loyola symposium. Hillsdale, N.J: Erlbaum, 1975. (a)

Posner, M. I., \& Snyder, C. R. R. Facilitation and inhibition in the processing of signals. In P. M. A. Rabbitt \& S. Dornic (Eds.), Attention and performance $V$. New York: Academic Press, 1975. (b)

ROEDIGER, H. L. Inhibition in recall from cuing with recall targets. Journal of Verbal Learning and Verbal Behavior, 1973, 12, 644-657.

Roediger, H. L. Inhibiting effects of recall. Memory \& Cognition, 1974, 2, 261-269.

Roediger, H. L., Stellon, C. C., \& Tulving, E. Inhibition from part-list cues and rate of recall. Journal of Experimental Psychology: Human Learning and Memory, 1977, 3, 174-188.

Rosch, E. Cognitive representations of semantic categories. Journal of Experimental Psychology: General, 1975, 104, 192-233. (a)

Rosch, E. The nature of mental codes for color categories. Journal of Experimental Psychology: Human Perception and Performance, 1975, 1, 303-322. (b)

Rundus, D. Negative effects of using list items as recall cues. Journal of Verbal Learning and Verbal Behavior, 1973, 12, 43-50.

Schvaneveldt, R. W., \& Meyer, D. E. Retrieval and comparison processes in semantic memory. In S. Kornblum (Ed.), Attention and performance IV. New York: Academic Press, 1973.
ShafFer, L. H. Multiple attention in continuous verbal tasks. In P. M. A. Rabbitt \& S. Dornic (Eds.), Attention and performance $V$. New York: Academic Press, 1975.

Shallice, T., \& Warrington, E. K. Word recognition in a phonemic dyslexic patient. Quarterly Journal of Experimental Psychology, 1975, 27, 187-199.

Shiffrin, R. M., \& Schneider, W. Controlled and automatic human information processing: II. Perceptual learning, automatic attending, and a general theory. Psychological Review, $1977,84,127-190$.

Stamecka, N. J. An examination of trace storage in free recall. Journal of Experimental Psychology, 1968, 76, 504-513.

Slamecka, N. J. Testing for associative storage in free recall. Journal of Experimental Psychology, 1969, 81, 557-560.

Sperling, G. The information available in brief visual presentations. Psychological Monographs, 1960, 74(Whole No. 498).

Stroop, J. R. Studies of interference in serial verbal reactions. Journal of Experimental Psychology, 1935, 18, 643-662.

Tulving, E., \& Pearlstone, I. Availability versus accessibility of information in memory for words. Journal of Verbal Learning and Verbal Behavior, 1966, 5, 381-391.

Watkins, M. J. Inhibition in recall with extralist "cues." Journal of Verbal Learning and Verbal Behavior, 1975, 14, 294-303.

(Received for publication January 11, 1979; revision accepted May $8,1979$. ) 